\title{
Hemorrhage in the Setting of Acute Severe Refractory Immune Thrombocytopenic Purpura: A Case Report
}

\section{Emily J Bryer*, Karla Asturias and Paul Kinniry \\ Department of Internal Medicine, University of Pennsylvania, USA}

*Corresponding author: Emily Jane Bryer, Department of Internal Medicine, Pennsylvania Hospital, University of Pennsylvania, Philadelphia PA, USA 19107, Fax:

2158293309; Tel: 2675814166; Email: Emily.bryer@pennmedicine.upenn.edu

\section{Case Report}

Volume 3 Issue 2

Received Date: August 13, 2019

Published Date: August 21, 2019

DOI: $10.23880 / \mathrm{hij}-16000143$

\section{Abstract}

Immune thrombocytopenic purpura (ITP) is a hematologic disorder characterized by platelet-directed autoantibodies that result in thrombocytopenia via splenic consumption. Patients with ITP usually have a platelet count $<100,000 \mathrm{u} / \mathrm{L}$, with lower counts associated with more severe ITP. While ITP can have a mild clinical spectrum with minor bruising and petechiae, it may also be associated with potentially fatal pulmonary or cerebral hemorrhage. This manuscript presents a comprehensive review of the pathophysiology, diagnosis, and current therapeutic strategies currently available to treat ITP. We present a case of severe and acute refractory ITP in the setting of persistent multifocal bleeding and a platelet count that eventually responded following eight distinct therapies.

Keywords: Systemic lupus erythematosus; Thrombocytopenia; Immune thrombocytopenic purpura; Gastrointestinal bleeding;Partial splenic embolization

Abbreviations: ITP: Immune Thrombocytopenic Purpura; SLE: Systemic Lupus Erythematosus; IVIG: Infusions of Intravenous Immunoglobulin; PRES: Posterior Reversible Encephalopathy Syndrome; APCs: Antigen-Presenting Cells; TTP: Thrombotic Thrombocytopenic Purpura; HUS: Hemolytic Uremic Syndrome.

\section{Case Report}

A 30-year-old woman with a past medical history of hypertension, systemic lupus erythematosus (SLE), chronic kidney disease in the setting of stage III lupus glomerulonephritis, and provoked venous thromboembolism presented with a one-month history of vaginal bleeding followed by gingival bleeding, hematuria, fatigue, and dyspnea. She was found to have a platelet count of 2 thousand/uL and hemoglobin of $4 \mathrm{~g} / \mathrm{dL}$. ADAMTS13 was negative and there was no evidence of schistocytes on peripheral blood smear. The patient was diagnosed with acute severe immune thrombocytopenic purpura.

The patient received daily solumedrol and three infusions of intravenous immunoglobulin (IVIG) without a response in her platelet count. In the setting of active bleeding with severe thrombocytopenia and anemia, she received multiple transfusions of platelets and red blood cells without appropriate response. The patient then developed acute respiratory distress syndrome with 
diffuse alveolar hemorrhage in the setting of suspected transfusion-related acute lung injury and/or transfusionassociated circulatory overload. She developed worsening oropharyngeal bleeding and required intubation for acute hypoxic respiratory failure. Family history was remarkable for a mother with SLE. The patient consumed two alcoholic drinks per week and denied drugs and tobacco. Outpatient medications included mycophenolate mofetil and hydroxychloroquine, both for SLE.

Upon arrival to our hospital, vital signs were all within normal limits. Labs showed leukocytes $44.67 \mathrm{uL}$ with $95 \%$ neutrophils, $2 \%$ lymphocytes, hemoglobin $7.1 \mathrm{~g} / \mathrm{dL}$ (MCV 88 ) with $10.5 \%$ reticulocytes, and platelets $2000 \mathrm{u} / \mathrm{L}$, creatinine $1.64 \mathrm{mg} / \mathrm{dL}$ (baseline 0.818 months prior) with unremarkable liver function tests. INR 1.1, PTT 27.6 seconds, fibrinogen $416 \mathrm{mg} / \mathrm{dL}$, dilute Russell's viper venom test elevated at 47 seconds. Peripheral smear showed absent platelets and 0-2 schistocytes per highpower-field (Figure 1). Rheumatologic labs showed dsDNA $150 \mathrm{IU} / \mathrm{mL}$, normal beta 2 glycoprotein, negative anticardiolipin antibody, positive anti-nuclear antibody, cyclic citrulline peptide antibody $8.1 \mathrm{U} / \mathrm{ml}, \mathrm{C} 369 \mathrm{mg} / \mathrm{dL}$, C4 $11 \mathrm{mg} / \mathrm{dL}$, dsDNA antibody 48, SS-A/Ro antibody positive with index 1.4, and RNP 3.3 with positive antibody. DAT and blood cultures from admission were both negative. EBV anti-nuclear antibody was 3.7, HIV was nonreactive, and $\mathrm{H}$. pylori antigen was negative. She was noted to have coarse breath sounds bilaterally with diffuse crackles, petechiae on bilateral tibia, and oozing from her right brachial arterial line. CXR with bilateral alveolar infiltrates consistent with acute respiratory distress syndrome (Figure 2).

\begin{tabular}{|l|l|}
\hline \\
Figure $1:$ Peripheral smear on admission: Absent & Figure 2: Peripheral smear on day 52: Interval increase \\
platelets, 0-2 schistocytes per hpf. & in platelets, now with giant platelets present. \\
\hline Interventions in Chronological Order of \\
Initiation
\end{tabular}

Table 1: A summary of all treatment therapies is available. 
Despite receipt of methylprednisolone and platelet transfusions, the patient continued to bleed from intravenous access sites and after accuchecks. She was started on rituximab (weekly x 4 weeks) and romiplostim (weekly x 6 weeks) without significant change in her platelet count. She subsequently had two sequential partial splenic embolizations with an estimated 15\% splenic function remaining intact without an improvement in platelet count. She underwent plasmapheresis with 10 units of fresh frozen plasma for three days followed by four weekly doses of eculizumab (Table 1).

Following splenic embolization and in the setting of eculizumab treatment, she received Ceftriaxone for meningococcal prophylaxis and atovaquone for Pneumocystis jirovecii prophylaxis. In the setting of vaginal bleeding, she was given leuprolide for menstrual suppression. The patient's course was complicated by serotonin syndrome, acute renal failure, Candida albicans fungemia, posterior reversible encephalopathy syndrome (PRES), hypertension, and melena found to be secondary to diffuse mucosal petechiae in the stomach requiring massive transfusion protocol. After four weeks of profound thrombocytopenia with single digit platelets and numerous interventions, her platelet counts gradually improved (Figure 2). A summary of all treatments is below (Figure 3).

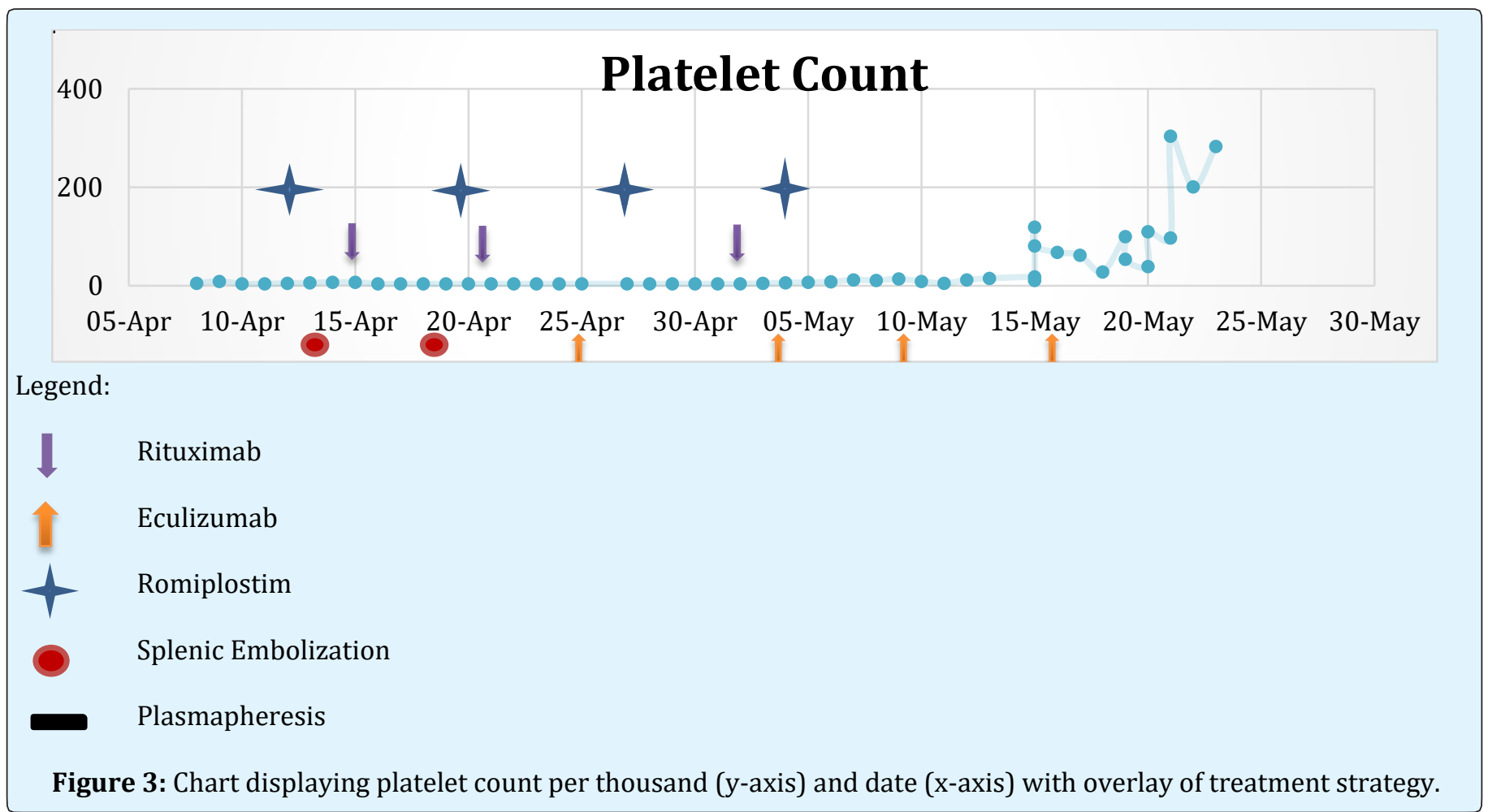

\section{Immune Thrombocyopenic Purpura}

Immune thrombocytopenic purpura (ITP) is an immune-mediated and acquired hematologic disorder characterized by a transient or persistent isolated decrease in platelet counts to less than $100 \times 109 / \mathrm{L}$ [1] . The production of antiplatelet autoantibodies accelerate platelet destruction via the antigen-presenting cells (APCs) of the spleen by opsonization, monocytes, macrophages, and T-cells [2,3]. ITP may occur with infections, malignancies, drugs, and immunodeficiencies and autoimmune diseases including systemic lupus erythematosus, Sjögren's syndrome, Evans syndrome, and antiphospholipid syndrome [3-5].

Symptoms of mild ITP include bruising and petechiae following minor trauma. Patients with platelet counts below $20,000 \mathrm{~mm}$ often present with oropharyngeal and gastrointestinal bleeding [4]. Severe cases of ITP may manifest as intra-pulmonary and intra-cerebral 
hemorrhage and can be fatal $[4,6]$. The diagnosis of ITP may include the detection of autoantibodies against platelet antigens [2]. However, platelet antibodies are only detected $60 \%$ of patients which may be a reflection of the limited sensitivity of the assay, the presence of unknown antigens, or additional mechanisms involved in platelet loss [7]. Due to the aforementioned limitations, most guidelines do not recommend routine testing of antiplatelet antibodies for the diagnosis of ITP. Instead, the diagnosis is made by a combination of a history, physical exam, complete blood count, evaluation of a peripheral blood smear, and the exclusion of other causes of thrombocytopenia. Some additional tests include the blood group, Rh, direct antiglobulin test, Helicobacter pylori, HIV, and HCV testing [8-10].

The objective of ITP therapy is to maintain a safe, but not necessarily normal, platelet count to ensure an acceptable quality of life with minimal treatment-related toxicity [1]. Treatment of ITP is typically reserved for patients who are bleeding and the platelet count threshold to warrant treatment remains controversial. Although most guidelines recommend treating adults with platelets less than $30,000 / \mathrm{mm}^{4}$, others recommend only treating if patients with clinical bleeding $[8,9,11]$. The discrepancy between guidelines necessitate the personalization of ITP therapeutics. Corticosteroids are the standard initial intervention in patients with ITP in the absence of critical hemorrhage. In the setting of active bleeding, and for individuals refractory to therapy with corticosteroids, Anti-D immunoglobulin [12] or intravenous immunoglobulin (IVIG) are generally recommended, either alone or in combination with steroids [7].

Splenectomy is a second-line treatment in patients with ITP however, despite response rates of around $80 \%$, it is associated with increased risks of infection, severe sepsis, and thrombosis [13]. Partial splenic embolization avoids most of the complications and limitations associated with a surgical splenectomy and should be considered if the latter is contraindicated [14]. Partial splenic embolization is a safe and effective alternative therapy for patients with steroid-resistant ITP, achieving a response in approximately $80 \%$ of patients [15]. Vaccination following splenectomy is critically important as these patients are at risk of infection from encapsulated organisms.

Other second-line treatments of ITP include rituximab, a monoclonal antibody directed at CD20, with an initial response rate between 40 to $60 \%$. Unfortunately, evidence is limited for sustained responses beyond 6-12 months $[16,17]$. More recent therapies for ITP include the thrombopoietin-receptor agonists, such as romiplostim and eltrombopag, which are effective among highly refractory and multiply treated patients with a response rate of up to $75 \%$ [18]. Eculizumab, a monoclonal antibody directed against CD5, is efficacious in treatment of thrombotic thrombocytopenic purpura (TTP), [19] however there is a paucity of data regarding its role in treatment of ITP. Similarly, while the removal of plateletdirected antibodies by plasmapheresis is standard of care in TTP, data is limited concerning its value as a treatment of ITP.

\section{Case Discussion}

We present an unusual and challenging case of acute severe ITP in the setting of active hemorrhage. In the absence of an identified acute viral precipitant, infection, or malignancy, SLE was suspected to trigger the development of acute ITP. It remains unclear what environmental or genetic factors predict the severity of ITP. Unfortunately, the refractory nature of this patient's thrombocytopenia increased her risk of hemorrhage. The presence of concomitant pulmonary, gastrointestinal, and vaginal hemorrhage in the setting of poor thrombotic function posed a unique therapeutic challenge. Aminocaproic acid helped to control the hemorrhage until her platelet counts increased to aid in hemostasis. Leuprolide, a gonadotropin-releasing hormone agonist, was selected to suppress vaginal bleeding with encouraging result.

Decisions regarding interventions, surgeries, and therapies require the individualization of treatment guidelines to opt for the presumed most efficacious option that carries the lowest risk. In this case, although the steroids theoretically should have treated both the ITP and the SLE, the platelet count did not respond. Following steroids, the patient received rituximab and romiplostim. The specific efficacy of these agents is difficult to precisely identify given both overlapping administration with other agents as well as the delayed onset of anticipated improvement. In the setting of persistent active bleeding and poor hemostatic function, splenectomy was considered; however, the patient's single-digit platelet count precluded her as a surgical candidate.

A total of eight distinct therapies were utilized in an effort to improve the platelet count. These therapies included: platelet transfusions, steroids, IVIG, rituximab, 
romiplostim, partial splenic embolization, plasmapheresis, and eculizumab. Of note, platelets were only administered in the setting of concomitant bleeding and were not transfused as an isolated attempt to solely improve platelet count. Eculizumab was trialed in efforts to treat this case as atypical hemolytic uremic syndrome (HUS) which may have been efficacious in improving platelet counts. While we cannot determine which single or combination of therapies was effective, the patient's platelet count did eventually increase after thirty days of treatment. This case emphasizes the necessity of further research to clarify the predictors of response to specific treatments, the optimal sequence of therapies in highly refractory cases, and the safety of therapy combinations.

\section{Conclusion}

ITP is a challenging hematologic disorder with a variety of pathologic mechanisms and varying response to therapies. While there are universal standardized guidelines for the treatment of ITP, therapy must be tailored to each patient. Current therapies include the use of steroids, intravenous immunoglobulin, monoclonal antibodies, thrombopoietin-receptor agonists and, in severe cases, splenectomy or partial splenic embolization. Future directions include the creation of precise tests and assays for the diagnosis of ITP as well as the development of additional therapeutic agents to lead to rapid improvement of platelet counts.

\section{Practice Points}

- ITP has a wide clinical spectrum related to platelet counts, ranging from minor bruising and bleeding, to potentially life-threatening hemorrhage

- ITP is associated with several infections, malignancies, drugs, immunodeficiencies and autoimmune conditions

- The diagnosis of ITP is made by a combination of a history, physical examination, and the exclusion of other causes of isolated thrombocytopenia

- Guidelines recommend treatment for ITP either with platelet counts less than $30,000 \mathrm{u} / \mathrm{L}$ or in the presence of active clinical bleeding

- First-line treatments include corticosteroids, IVIG and anti-D immunoglobulin

- Second-line treatments include splenectomy, partial splenic embolization, rituximab and TPO-RA

\section{References}

1. Rodeghiero F, Stasi R, Gernsheimer T, Michel M, Provan D, et al. (2009) Standardization of terminology, definitions and outcome criteria in immune thrombocytopenic purpura of adults and children: report from an international working group. Blood 113(11): 2386-2393.

2. Cines DB, Bussel JB, Liebman HA, Luning Prak ET (2009) The ITP syndrome: pathogenic and clinical diversity. Blood 113(26): 6511-6521.

3. Li J, Sullivan JA, Ni H (2018) Pathophysiology of immune thrombocytopenia. Curr Opin Hematol 25(5): 373-381.

4. Justiz Vaillant AA, Gupta N (2019) Thrombocytopenic Purpura Immune. StatPearls Publishing.

5. Audia S, Mahévas M, Samson M, Godeau B, Bonnotte B (2017) Pathogenesis of immune thrombocytopenia. Autoimmun Rev 16(6): 620-632.

6. Kayal L, Jayachandran S, Singh K (2014) Idiopathic thrombocytopenic purpura. Contemp Clin Dent 5(3): 410-414.

7. Stasi R, Evangelista ML, Stipa E, Buccisano F, Venditti A, et al. (2008) Idiopathic thrombocytopenic purpura: Current concepts in pathophysiology and management. Thromb Haemost 99(1): 4-13.

8. Neunert C, Lim W, Crowther M, Alan Cohen, Lawrence Solberg, et al. (2011) The American Society of Hematology 2011 evidence-based practice guideline for immune thrombocytopenia. Blood 117(16): 41904207.

9. Provan D, Stasi R, Newland AC, Blanchette VS, BoltonMaggs $P$, et al. (2010) International consensus report on the investigation and management of primary immune thrombocytopenia. Blood 115(2): 168-186.

10. Sugiura T, Yamamoto $K$, Murakami K, Horita $S$, Matsusue Y, et al. (2018) Immune Thrombocytopenic Purpura Detected with Oral Hemorrhage: a Case Report. J Dent (Shiraz, Iran). 19(2): 159-163.

11. British Committee for Standards in Haematology General Haematology Task Force (2003) Guidelines for the investigation and management of idiopathic thrombocytopenic purpura in adults, children and in pregnancy. Br J Haematol 120(4): 574-596.

12. Cheung E, Liebman HA (2009) Anti-RhD immunoglobulin in the treatment of immune thrombocytopenia. Biologics 3: 57-62. 
13. Thai LH, Mahévas $M$, Roudot-Thoraval F, Limal N, Languille L, et al. (2016) Long-term complications of splenectomy in adult immune thrombocytopenia. Medicine (Baltimore) 95(48): e5098.

14. Nakaseko C, Shimizu N, Sakaida E, Emi Togasaki, Masahiro Takeuchi, et al. (2013) Partial Splenic Embolization for the Treatment of Steroid-Resistant Chronic Idiopathic Thrombocytopenic Purpura. Blood 122(21): 3543.

15. Togasaki E, Shimizu N, Nagao Y, Kawajiri MC, Shimizu $\mathrm{R}$, et al. (2018) Long-term efficacy of partial splenic embolization for the treatment of steroid-resistant chronic immune thrombocytopenia. Ann Hematol 97(4): 655-662.
16. Cuker A, Cines DB, Neunert CE (2016) Controversies in the treatment of immune thrombocytopenia. Curr Opin Hematol 23(5): 479-485.

17. Chugh S, Darvish-Kazem S, Lim W, Crowther MA, Ghanima W, et al. (2015) Rituximab plus standard of care for treatment of primary immune thrombocytopenia: a systematic review and metaanalysis. Lancet Haematol 2(2): 75-81.

18. Shih A, Nazi I, Kelton JG, Arnold DM (2014) Novel treatments for immune thrombocytopenia. Presse Med 43(4): 87-95.

19. Chapin J, Weksler B, Magro C, Laurence J (2012) Eculizumab in the treatment of refractory idiopathic thrombotic thrombocytopenic purpura. Br J Haematol 157(6): 772-774. 